Review Article

\title{
Nitric Oxide Bioavailability in Obstructive Sleep Apnea: Interplay of Asymmetric Dimethylarginine and Free Radicals
}

\author{
Mohammad Badran, ${ }^{1}$ Saeid Golbidi, ${ }^{1}$ Najib Ayas, ${ }^{2,3,4}$ and Ismail Laher ${ }^{1}$ \\ ${ }^{1}$ Department of Pharmacology and Therapeutics, Faculty of Medicine, University of British Columbia, \\ Vancouver, BC, Canada V6T 1Z3 \\ ${ }^{2}$ Divisions of Critical Care and Respiratory Medicine, Department of Medicine, 2775 Laurel Street, 10th Floor, \\ University of British Columbia, Vancouver, BC, Canada V5Z 1M9 \\ ${ }^{3}$ Sleep Disorders Program, UBC Hospital, Room G-285, 2111 Wesbrook Mall, Vancouver, BC, Canada V6T $2 B 5$ \\ ${ }^{4}$ Division of Critical Care Medicine, Providence Health Care, St. Paul's Hospital, Vancouver, BC, Canada V6Z 1 Y6 \\ Correspondence should be addressed to Ismail Laher; ilaher@mail.ubc.ca
}

Received 6 February 2015; Revised 15 April 2015; Accepted 17 April 2015

Academic Editor: Giora Pillar

Copyright @ 2015 Mohammad Badran et al. This is an open access article distributed under the Creative Commons Attribution License, which permits unrestricted use, distribution, and reproduction in any medium, provided the original work is properly cited.

Obstructive sleep apnea (OSA) occurs in $2 \%$ of middle-aged women and $4 \%$ of middle-aged men and is considered an independent risk factor for cerebrovascular and cardiovascular diseases. Nitric oxide (NO) is an important endothelium derived vasodilating substance that plays a critical role in maintaining vascular homeostasis. Low levels of NO are associated with impaired endothelial function. Asymmetric dimethylarginine (ADMA), an analogue of L-arginine, is a naturally occurring product of metabolism found in the human circulation. Elevated levels of ADMA inhibit NO synthesis while oxidative stress decreases its bioavailability, so impairing endothelial function and promoting atherosclerosis. Several clinical trials report increased oxidative stress and ADMA levels in patients with OSA. This review discusses the role of oxidative stress and increased ADMA levels in cardiovascular disease resulting from OSA.

\section{Obstructive Sleep Apnea}

Obstructive sleep apnea (OSA) is a sleep-breathing disorder characterized by momentary episodes of either $80-100 \%$ reductions in airflow for periods of ten seconds or more (apnea) or $50-80 \%$ reductions (hypopnea) caused by a collapsed or obstructed upper airway; these two conditions can lead to hypoxemia (low levels of oxygen in blood) and hypercapnia (high levels of carbon dioxide in blood). Patients are categorized as having mild, moderate, or severe OSA depending on the apnea/hypopnea index (AHI), which is defined as the total numbers of obstructive apnea/hypopnea episodes per hour of sleep. In normal individuals the index is usually 5 or lower, while it is 5-15 in mild, $15-30$ in moderate, and 30 or more in severe OSA patients [1,2]. In patients with mild OSA the oxyhemoglobin saturation drops to $95 \%$ and can drop below $80 \%$ in severe cases. Obstruction of the airways results in greater breathing effort and a more negative intrathoracic pressure, resulting in arousal, sleep interruption, and reopening of the airways [3] as summarized in Figure 1.

Risk factors for sleep apnea include obesity, craniofacial abnormalities, smoking, male gender, short neck, and menopause in women. Obesity is one of the main risk factors of sleep apnea since $60 \%$ to $90 \%$ of OSA patients are obese and there is a positive correlation between body mass index (BMI) and OSA $[4,5]$. The overlap of obesity and OSA poses a challenge to ascribing the relative contributions of these comorbidities to cardiovascular complications. Greater adiposity and a shorter neck add weight to the soft tissue within the upper airway and the neck, so further increasing airway collapsibility [6]. The economic burden created by OSA is considerable; for example, OSA-related automobile collisions in the year 2000 alone are attributed to 1400 fatalities at a total cost of 15.9 billion dollars in the USA. It is estimated that treatment with continuous positive airway 


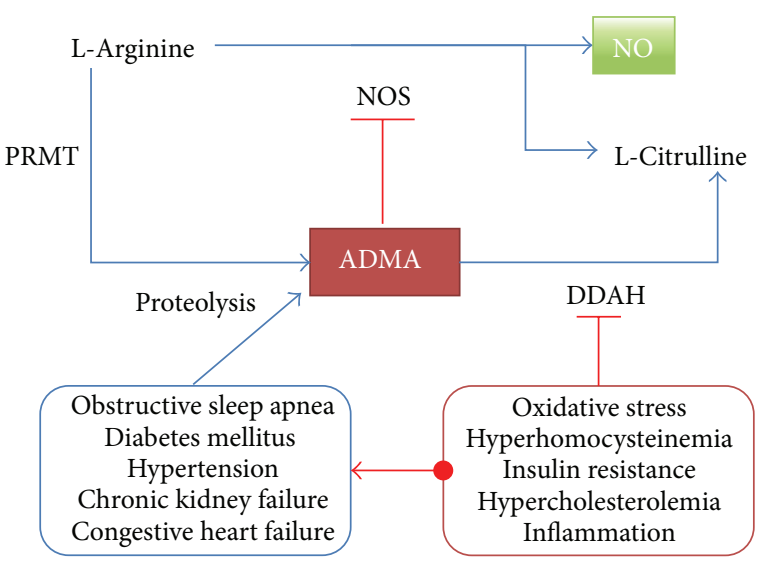

FIGURE 1: Pathology of ADMA. ADMA: asymmetric dimethylarginine; DDAH: dimethylarginine dimethylaminohydrolase; NO: nitric oxide; NOS: nitric oxide synthase; PRMT: protein arginine methyltransferase.

pressure (CPAP) produced a saving of 7.9 billion dollars and 1000 lives [7].

Between 3.7\% and $26 \%$ of the population has an AHI above 5. The prevalence of OSA, defined mainly by AHI frequency and the presence of hypersomnolence, is estimated to range from $1.2 \%$ to $7.5 \%$. Due to the lack of homogeneity in these epidemiologic studies, estimates show wide variations. For instance, some studies were performed in preselected population groups while others included a high number of subjects who were suspected of having OSAS because of their snoring frequency. Moreover, some earlier studies did not include subjects over 60 years of age [8]. Unfortunately, most of those who are affected by OSA remain undiagnosed despite medical advances [9].

The results of several clinical studies strongly suggest that OSA is an independent risk factor for cardiovascular diseases such as hypertension, coronary artery disease, stroke, and heart failure [10-17]. Several mechanisms are thought to link OSA and vascular diseases, including increases in sympathetic activation, oxidative stress, inflammation, endothelial dysfunction, coagulation, and metabolic dysregulation [18]. We review the bioavailability of nitric oxide (NO) metabolism in OSA and the role of asymmetric dimethylarginine (ADMA) as a risk factor for endothelial dysfunction.

\section{ADMA and Nitric Oxide Metabolism}

The 1998 Nobel Prize in Medicine and Physiology was awarded for the discovery of NO as a signaling molecule in the cardiovascular system [19]. This gaseous vasodilator has a half-life of 2-30 sec [20] and is synthesized from the amino acid L-arginine in endothelial cells by the calciumcalmodulin dependent enzyme nitric oxide synthase (NOS) $[21,22]$. It was later found that NO relaxes smooth muscles despite the high levels of calcium and activated myosin, most likely via NO-mediated heat shock protein 20 (HSP20) phosphorylation $[22,23]$. NO diffuses in blood where it binds to hemoglobin and is then excreted in urine as nitrate after being oxidized. Vasodilation is produced when NO stimulates soluble guanylate cyclase, leading to increased production of cyclic guanosine monophosphate (GMP) that activates GMP-dependent kinases to decrease intracellular calcium concentrations [24]. NO is also antithrombotic, antiproliferative, and anti-inflammatory [25-33].

ADMA is a naturally occurring L-arginine analog derived from the proteolysis of methylated proteins [34]. The terminal guanidine group of arginine is demethylated by two classes of protein arginine methyltransferases (PRMTs). Type 1 PRMTs catalyze the formation of ADMA, whereas type 2 PRMTs lead to the formation of symmetric dimethylarginine (SDMA) [35]. ADMA inhibits all three isoforms of nitric oxide synthase (NOS) producing NO (neuronal NOS "nNOS," inducible NOS "iNOS," and endothelial NOS "eNOS") but with different affinities; for example, ADMA has an $\mathrm{IC}_{50}$ value of $10 \mu \mathrm{mol} / \mathrm{L}$ in cultured endothelial cells [36] but low affinities for $\mathrm{nNOS}$ and iNOS $\left(K_{i}>300 \mu \mathrm{mol} / \mathrm{L}\right)$ [37]. ADMA is both exported from its site of origin (such as liver, kidney, or lung) and imported from the plasma at distant sites via cationic amino acid transporters (CATs) in exchange for arginine and other cationic amino acids (CAAs) [38]. While ADMA is present in the liver and kidneys, the lungs produce 4 times more $[39,40]$. ADMA competes with Larginine for the binding site in the active center of NOS enzymes [41]. Furthermore, ADMA can "uncouple" NOS by shifting the balance from NO generation to superoxide production such as formation of reactive oxygen species (ROS) [42]. The role of SDMA in the endothelial NO pathway remains unclear. SDMA and ADMA are able to interfere with the substrate availability of NOS by inhibiting the transmembrane cationic amino acid transport (CAT) system of L-arginine, but the $\mathrm{IC}_{50}$ values (ADMA and SDMA inhibited CAT1-mediated uptake of L-arginine with $\mathrm{IC}_{50}$ values of 758 (460-1251) $\mu \mathrm{mol} / \mathrm{L}$ and 789 (481-1295) $\mu \mathrm{mol} / \mathrm{L}$, resp.) are above the estimated endogenous ADMA and SDMA concentrations $(13.5 \pm 0.13 \mu \mathrm{mol} / \mathrm{L})$ [43]. Almost $80 \%$ of ADMA is enzymatically hydrolyzed by dimethylarginine dimethylaminohydrolase (DDAH), which is expressed in two isoforms: DDAH-1 and DDAH-2, which have distinct tissue distribution and are encoded by different genes and, possibly, exhibit distinct functional roles $[44,45]$. The $K_{m}$ of DDAH is approximately $180 \mu \mathrm{mol} / \mathrm{L}$ and is much higher than normal intracellular concentrations, which enables the enzyme to properly accomplish this task. This ensures that under normal conditions the enzyme performs in the linear part of the substrate-velocity curve, meaning that the rate of ADMA degradation is approximately proportional to its concentration. The activity of DDAH increases with higher ADMA levels, thus preventing accumulation of ADMA. The high $K_{m}$ of DDAH prevents accumulation of high ADMA levels and ensures sufficient amounts of ADMA are available to function as a regulator of NOS activity [46].

Elevated ADMA levels occur in patients with chronic kidney failure and were proposed to act as possible endogenous inhibitors of NO synthesis by Vallance et al. [47]. Plasma levels of ADMA predicted all causes of mortality, but not cardiovascular disease incidence, in the Framingham Offspring Study of 3320 normal subjects followed up for 11 
TABLE 1: ADMA as a cardiovascular risk marker in some cardiovascular clinical studies.

\begin{tabular}{|c|c|c|c|}
\hline CVD & AMDA levels $(\mu \mathrm{mol} / \mathrm{L})$ & Result & Reference \\
\hline Stable CAD & $>0.62$ (highest quartile) & $\begin{array}{l}\text { 3.9-fold increased risk of acute coronary } \\
\text { disease }\end{array}$ & {$[58]$} \\
\hline MI & $\begin{array}{l}1.45 \text { (median range of the } \\
\text { highest tertile) }\end{array}$ & ADMA predicts mortality after MI & {$[59]$} \\
\hline $\mathrm{CHF}$ & $>2.5$ (highest tertile) & $\begin{array}{l}\text { High ADMA levels are associated with } \\
\text { cardiac decompensation and major } \\
\text { adverse cardiovascular events }\end{array}$ & {$[60]$} \\
\hline $\mathrm{AF}$ & $>0.63$ (cut-off value) & $\begin{array}{l}\text { High ADMA levels predict AF recurrence } \\
\text { after catheter ablation }\end{array}$ & {$[52]$} \\
\hline Ischemic stroke & $\begin{array}{l}>1.43 \text { (above } 90 \text { th } \\
\text { percentile) }\end{array}$ & $\begin{array}{l}\text { High ADMA levels are associated with } \\
\text { increased risk of stroke in old patients } \\
\text { and in patients with } \\
\text { hyperhomocysteinemia }\end{array}$ & {$[54]$} \\
\hline
\end{tabular}

ADMA: asymmetric dimethylarginine; AF: atrial fibrillation; CAD: coronary artery disease; CHF: congestive heart failure; MI: myocardial infarction.

years [48]. The many studies on ADMA have led many to propose that it alters NO biosynthesis and mediates several cardiovascular complications as summarized in Table 1 [49]. ADMA levels are elevated in patients with hypertension [50], diabetes mellitus [51], coronary artery disease [21], arrhythmias [52], heart failure [53], and cerebrovascular disease [54].

Bae et al. measured plasma ADMA levels in 48 patients newly diagnosed with acute coronary syndrome (ACS) and followed changes in ADMA concentrations during their medical therapy which included a combination of drugs with or without percutaneous coronary interventions. Concentrations of plasma ADMA in ACS patients were double that in age-matched control patients, but levels decreased significantly after 2 weeks of treatment [55]. Levels of ADMA were also significantly elevated in 198 hemodialysis patients with left ventricular hypertrophy $(\mathrm{LVH})(3.00 \mu \mathrm{mol} / \mathrm{L}$ with $\mathrm{LVH}$ and $1.88 \mu \mathrm{mol} / \mathrm{L}$ without $\mathrm{LVH}$ ) and were correlated with left ventricular mass $(r=0.26, P<0.001)$ [56]. Levels of ADMA are also increased after cardioembolic infarction and transient ischemic attack (TIA) in 363 CVD patients when compared to 48 control patients [57]. Results of other studies on ADMA in CVD are discussed in Table 1.

\section{ADMA in OSA}

It is now well established that OSA is a cardiovascular risk factor and that ADMA has the potential to exacerbate cardiovascular disease. But does OSA influence ADMA levels? Many studies show increases in ADMA levels in OSA patients; Barceló et al. measured plasma ADMA in 23 OSA patients and found it to be significantly higher $(1.17 \mu \mathrm{mol} / \mathrm{L})$ compared to control $(0.87 \mu \mathrm{mol} / \mathrm{L}, P<0.01)$ [61]. Ozkan et al. also reported nonsignificant increases in ADMA levels in OSA patients [62]. Treatment with CPAP for 4 weeks lowered ADMA levels in patients with OSA while also improving forearm mediated dilation (FMD) $(0.22 \pm 0.27 \mathrm{~mol} / \mathrm{L}$ before $\mathrm{CPAP}, 0.21 \pm 0.44 \mathrm{~mol} / \mathrm{L}$ at 1 week, and $0.16 \pm 0.27 \mathrm{~mol} / \mathrm{L}$ at 4 weeks after CPAP, $P=0.054$ ) [63]. It is unclear how OSA increases ADMA levels but it is important to note that CPAP treatment can decrease its levels.

\section{Oxidative Stress in OSA}

Reactive oxygen and nitrogen species (ROS, RNS) are normal products of cellular metabolism labeled as molecules or molecular fragments. They contain one or more unpaired electrons in their molecular or atomic orbitals. At low concentrations, these species play an important role in cellular signaling, specifically in defense against infectious agents and induction of mitogenic response [64]. Overproduction of ROS/RNS or deficiency of enzymatic and nonenzymatic antioxidants can lead to oxidative and nitrosative stress. Consequently, oxidative stress leads to an imbalance between prooxidant/antioxidant reactions related to oxygen metabolism. ROS can oxidize lipids, protein, or DNA, so inhibiting their function and disturbing many cellular processes [64]. As a result, oxidative stress has been implicated in many human diseases (e.g., cardiovascular diseases, cognitive impairment, and diabetes) [65-67] as well as in the aging process [68].

Intermittent hypoxia (IH), a hallmark of OSA, is characterized by repeated episodes of hypoxia interspersed with episodes of normoxia, potentially similar to the ischemia/ reperfusion $(I / R)$ that results in injury due to the burst of ROS production during the reperfusion period [69]. It is well established that oxidative stress is a major consequence of $I / R$ injuries [70]. Similarly, cycles of IH in OSA patients promote ROS generation and oxidative stress through comparable pathways and various sources, as described in the next section.

\section{Sources of ROS/RNS in OSA}

During aerobic respiration, approximately $3-5 \%$ of the oxygen consumed by the mitochondria is converted to superoxide anion, a major form of ROS. Due to hypoxia, there is an increase in ROS production as a result of excessive 
mitochondrial reduction [64,71, 72]. Numerous clinical and animal studies demonstrate that $\mathrm{IH}$ is correlated with mitochondrial dysfunction, leading to oxidative stress [7376]. Another important source of ROS in OSA is NADPH oxidase, primarily expressed in leukocytes and activated during inflammatory processes such as infections, where it produces superoxide anions to destroy pathogens. There is increased production of ROS in stimulated neutrophils and monocytes from OSA patients as shown by Schulz et al. [77]. Other studies, however, reported high ROS production even in nonstimulated neutrophils and monocytes [78, 79]. Recent studies by Loffredo et al. assessed FMD in children with OSA and found that it is lower when compared to control and it was inversely correlated with serum soluble NOX2-derived peptide (sNOX2-dp). After adenotonsillectomy, however, FMD was significantly increased and sNOX2-dp was significantly decreased. These data suggest that NOX2-derived oxidative stress is associated with arterial dysfunction in children with OSA [80]. In addition, animal studies show that NADPH oxidase is activated in many tissues such as the brain and carotid body in response to IH $[81,82]$. Other sources of ROS that have been extensively studied in IH and OSA are xanthine oxidase [83, 84] and uncoupled eNOS [85].

\section{Markers of Oxidative Stress in OSA}

Throughout the last decade, there has been accumulation of much evidence that linked OSA and IH to oxidative stress. Oxidative stress markers such as lipid peroxidation, protein carbonylation, and DNA oxidation are prominent in OSA patients and animals subjected to IH. Patients with severe OSA have higher urinary 8-isoprostane levels, which then decreased after 6 months of CPAP therapy [86]. In a different study, TBARS levels were significantly higher in severe OSA patients after overnight fasting when compared to control [87]. In contrast, Svatikova et al. measured TBARS, oxidized LDL, and isoprostanes in healthy OSA patients with no comorbidities and found no association between severity of OSA and oxidative stress markers [88]. They suggested that, in the absence of significant comorbidities, sleep apnoea does not, in and of itself, initiate the generation of oxidative stress or lipid peroxidation. However, it is possible that, in the setting of comorbidities such as hypertension, vascular disease, and the metabolic syndrome, the oxidative consequences of sleep apnoea may become apparent. In another study, Vatansever et al. measured protein carbonyl and adiponectin levels in patients with mild, moderate, and severe OSA. They found that adiponectin levels were significantly decreased while protein carbonyl levels were significantly elevated in moderate to severe OSA patients but not in mild OSA ones when compared to control [89]. Yamauchi et al. measured another marker of oxidative related DNA damage, 8-OHdG, in OSA patients. They compared 8-OHdG levels between patients with nonsevere OSA $(\mathrm{AHI}<30)$ and patients with severe OSA (AHI > 30) and found that it was higher in severe OSA patients. They also found that $8-\mathrm{OHdG}$ is significantly correlated with AHI, oxygen desaturation index (ODI), and duration of oxygen saturation $<90 \%$. However, after adjusting for confounding factors, only ODI was significantly correlated to ODI [90].

\section{Endothelial Dysfunction in OSA}

Endothelial dysfunction manifests as an imbalance between vasodilating and vasoconstricting substances produced by or acting on the endothelium. It is clinically relevant in OSA even in subjects with no history of vascular disease or comorbidities [91]. Decreased endothelium-dependent vasodilation has been reported in several studies using FMD. For example, Kato et al. reported that OSA patients had blunted response to acetylcholine while responses to sodium nitroprusside or verapamil were unaltered [92]. Other studies analyzed nitrite/nitrate levels as a measure of circulating NO levels and reported reductions in OSA subjects [91, 93]. However, CPAP treatment of OSA patients restores circulating NO levels and the levels of its substrate L-arginine [94] as well as FMD [95].

Suggested mechanisms for endothelial dysfunction include (1) interaction between $\mathrm{NO}$ and superoxide anion leading to increased formation of a highly unstable RNS peroxynitrite, (2) decreased expression and/or uncoupling of endothelial nitric oxide synthase (eNOS), and (3) increased levels of endogenous eNOS inhibitors such as ADMA [1]. The large volume of distribution and short half-life of peroxynitrite could account for similar levels of nitrotyrosine in OSA and healthy subjects [96, 97]. Microcirculatory endothelial cells (EC) from OSA patients have increased formation of peroxynitrite [98]. In cultured endothelial cells from OSA patients, Jelic and le Jemtel reported a significant decrease in both total and phosphorylated eNOS levels, which were restored by CPAP treatment [99]. Moreover, Tanaka et al. suggested that eNOS activation is regulated by redox status and that increased oxidative stress reduced eNOS activity by suppressing its phosphorylation [100]. Nonetheless, the expression and activity of eNOS have been reported to be upregulated, $[101,102]$ downregulated, $[103,104]$, or unchanged [105] in various experimental models of hypoxia and repetitive hypoxia/reoxygenation. Interestingly, Kaczmarek et al. showed that cultured endothelial cells originating from distinct vascular beds in OSA patients and mice responded differently to $\mathrm{IH}$ stress in terms of eNOS expression [106]. Another cause of endothelial impairment is the eNOS uncoupling. For production of $\mathrm{NO}$ to occur, five cofactor groups (FAD, FMN, heme, $\mathrm{Ca}^{2+}$-calmodulin, and $\mathrm{BH}_{4}$ ) are needed to incorporate oxygen in L-arginine. A lack of any of these cofactors leads to production of superoxide anion instead of $\mathrm{NO}$ and adds insult to injury [18]. For instance, increased ROS production, especially superoxide anion during hypoxia, can lead to the oxidation of $\mathrm{BH}_{4}$ to $\mathrm{BH}_{2}$ rendering eNOS in an uncoupled state as shown by Antoniades et al. [107]. They also reported that increased levels of arginase II degrade L-arginine, leading to further eNOS uncoupling. A recent study by Varadharaj et al. reported that eNOS dysfunction in OSA patients was reversible with $\mathrm{BH}_{4}$ treatment [108]. 


\section{ADMA in OSA}

A recent meta-analysis of 22 studies with a total of 6168 patients reported a correlation between ADMA levels and carotid intima-media thickness (pooled correlation coefficient of $0.29 ; P<0.001$ ) [109]. This relation was stronger in patients with chronic kidney disease (CKD) than in subjects with normal kidney function. Patients with essential hypertension have impaired FMD and increased serum ADMA levels when compared to controls $(0.59 \pm 0.14 \mu \mathrm{mol} / \mathrm{L}$ versus $0.40 \pm 0.09 \mu \mathrm{mol} / \mathrm{L}, P<0.0001)$; these measures independently accounted for $33.9 \%$ of the interindividual variability in peak FMD [110]. In another cross-sectional study of 121 nondiabetic patients with proteinuria, ADMA levels were independently related to FMD $(>2.76 \mu \mathrm{mol} / \mathrm{L}$; $\left.r^{2}=0.27, P=0.0002\right)$ and were correlated with both proteinuria $\left(>2.5 \mathrm{~g} / \mathrm{d} ; r^{2}=0.40, P<0.001\right)$ and the presence or absence of secondary amyloidosis $\left(r^{2}=0.42, P=0.0003\right)$ [111]. The Cardiovascular Risk in Young Finns Study, a followup study of cardiovascular risk from childhood to adulthood, used ultrasound to measure FMD both in 2001 and 2007 in 1808 healthy subjects aged 24-39 years at baseline. Using a multivariable model adjusted with brachial diameter and conventional cardiovascular risk factors, the study reported that baseline ADMA levels were inversely associated with FMD measured six years later $(\beta \pm$ SE: $-1.89 \pm 0.69 \%, P=$ 0.006). This data suggests that plasma ADMA can predict endothelial function in subjects with no atherosclerotic disease, suggesting that ADMA may be a useful biomarker of endothelial dysfunction and atherosclerosis progression [112], as illustrated in Figure 2. OSA and COPD are among the most common pulmonary diseases, such that many patients have both disorders; this "overlap syndrome" causes more severe nocturnal hypoxemia than either disease alone. ADMA levels were measured in these patients before and after CPAP and there was no significant difference $(0.58 \pm$ 0.10 versus $0.61 \pm 0.12, P=0.32$ ) despite the reduction in serum levels of other inflammatory factors such as CRP $(0.83 \pm 0.95$ versus $0.53 \pm 0.56, P=0.02)[76,113]$. Barcelo et al. investigated the influence of OSA on the diurnal variations in some markers of endothelial dysfunction such as ADMA and soluble CD40 ligand and found that ADMA levels were significantly related to arousal index $(P=0.046)$. They also suggested that ADMA levels might be dependant either on obesity index or metabolic dysfunction rather than on OSA alone [114]. In nonobese children with OSA, Gozal et al. reported no differences in ADMA levels compared to matched controls $[0.79 \pm 0.20 \mu \mathrm{mol} / \mathrm{L}$ in OSA children $(n=46)$ and $0.87 \pm 0.19 \mu \mathrm{mol} / \mathrm{L}$ in controls $(n=22$; $p=\mathrm{NS})$ ]. Soluble CD40 ligand levels were higher in OSA compared with controls $(15,128 \pm 597 \mathrm{pg} / \mathrm{mL}$ versus $5729 \pm$ $653 \mathrm{pg} / \mathrm{mL}, P<0.00001)$ and were reduced after treatment (adenotonsillectomy) $(9866 \pm 702 \mathrm{pg} / \mathrm{mL}, P<0.0002)$ [115].

\section{ADMA as a Treatment Target}

Treatment with CPAP decreases ADMA levels and improves endothelial function. Another option would be supplementation with L-arginine, since it can displace ADMA from its

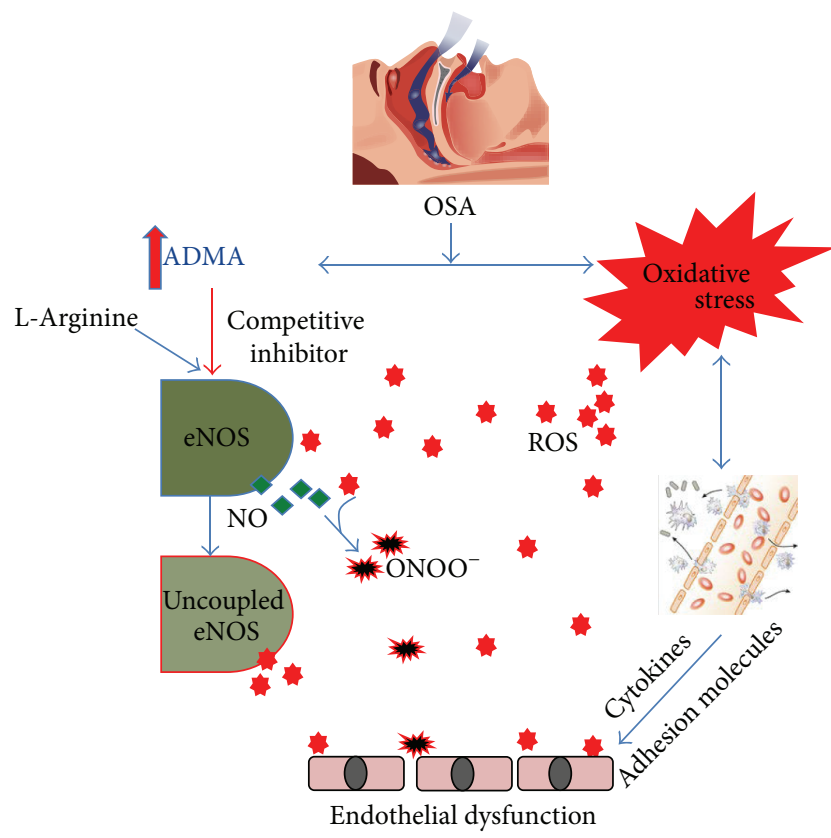

FIgure 2: Oxidative stress and high ADMA levels can cause endothelial dysfunction in OSA. ADMA: asymmetric dimethylarginine; eNOS: endothelial nitric oxide synthase; NO: nitric oxide; $\mathrm{ONOO}^{-}$: peroxynitrite; OSA: obstructive sleep apnea; ROS: reactive oxygen species.

receptor and also improve NO bioavailability despite the fact that its plasma concentration is 25 times in excess of it's $K_{m}$ for eNOS $[36,116]$. Unfortunately, L-arginine is ineffective after long-term use and, even worse, is correlated with mortality when given to post-MI patients [117, 118]. Other drugs used to lower ADMA levels include statins, angiotensin converting enzyme inhibitors (ACEI), $\beta$-blockers, aspirin, and antioxidants such as vitamins $E$ and $C$. These have largely been used for short periods in small groups of patients [119]. Largescale clinical trials are needed to evaluate the usefulness of these drugs in OSA patients. The therapeutic regulation of ADMA via DDAH is another possible mechanism to increase NO bioavailability $[120,121]$. The clinical relevance of regulating the endothelial ADMA/NO pathway in patients with OSA and oxidative stress induced by ADMA may be a useful strategy pending the availability of selective DDAH regulators.

\section{Conclusion}

There is extensive support for the role of ADMA as a regulator of NO production by the vascular endothelium. Data from cell culture and animal experiments and crosssectional studies in humans suggest an association between elevated ADMA concentrations and cardiovascular diseases. Elevation of ADMA levels, possibly in combination with increases in superoxide levels in patients with OSA, reduces the bioavailability of NO and leads to endothelial dysfunction to promote atherosclerosis and cardiovascular disease. Prospective clinical studies suggest that ADMA may be 
a potential diagnostic tool for cardiovascular risk assessment, but the use of ADMA in currently applied risk scores remains untested.

To date, pharmacological strategies targeting ADMA in cardiovascular risk reduction have been disappointing, as most lipid lowering and blood pressure-lowering drugs did not change ADMA concentrations significantly. Currently, only treatment with CPAP has been shown to normalize ADMA levels in OSA patients. Specific therapeutic interventions of DDAH/ADMA metabolism are needed to perform randomized controlled trials to assess the clinical benefits of modulating ADMA levels in OSA patients.

\section{Conflict of Interests}

The authors declare that there is no conflict of interests regarding the publication of this paper.

\section{References}

[1] M. Badran, N. Ayas, and I. Laher, "Insights into obstructive sleep apnea research," Sleep Medicine, vol. 15, no. 5, pp. 485-495, 2014.

[2] S. Golbidi, M. Badran, N. Ayas, and I. Laher, "Cardiovascular consequences of sleep apnea," Lung, vol. 190, no. 2, pp. 113-132, 2012.

[3] K. Gleeson, C. W. Zwillich, and D. P. White, "The influence of increasing ventilatory effort on arousal from sleep," American Review of Respiratory Disease, vol. 142, no. 2, pp. 295-300, 1990.

[4] M. Badran, S. Golbidi, A. Devlin, N. Ayas, and I. Laher, "Chronic intermittent hypoxia causes endothelial dysfunction in a mouse model of diet-induced obesity," Sleep Medicine, vol. 15, no. 5, pp. 596-602, 2014.

[5] T. Young, P. E. Peppard, and D. J. Gottlieb, "Epidemiology of obstructive sleep apnea: a population health perspective," American Journal of Respiratory and Critical Care Medicine, vol. 165, no. 9, pp. 1217-1239, 2002.

[6] A. R. Schwartz, S. P. Patil, S. Squier, H. Schneider, J. P. Kirkness, and P. L. Smith, "Obesity and upper airway control during sleep," Journal of Applied Physiology, vol. 108, no. 2, pp. 430-435, 2010.

[7] N. Alghanim, V. R. Comondore, J. Fleetham, C. A. Marra, and N. T. Ayas, "The economic impact of obstructive sleep apnea," Lung, vol. 186, no. 1, pp. 7-12, 2008.

[8] S. Tufik, R. Santos-Silva, J. A. Taddei, and L. R. A. Bittencourt, "Obstructive sleep apnea syndrome in the Sao Paulo Epidemiologic Sleep Study," Sleep Medicine, vol. 11, no. 5, pp. 441-446, 2010.

[9] N. M. Punjabi, "The epidemiology of adult obstructive sleep apnea," Proceedings of the American Thoracic Society, vol. 5, no. 2, pp. 136-143, 2008.

[10] R. P. Pedrosa, L. F. Drager, C. C. Gonzaga et al., "Obstructive sleep apnea: the most common secondary cause of hypertension associated with resistant hypertension," Hypertension, vol. 58, no. 5, pp. 811-817, 2011.

[11] P. Lavie, P. Herer, and V. Hoffstein, "Obstructive sleep apnoea syndrome as a risk factor for hypertension: population study," British Medical Journal, vol. 320, no. 7233, pp. 479-482, 2000.

[12] T. Konecny, F. H. Sert Kuniyoshi, M. Orban et al., "Underdiagnosis of sleep apnea in patients after acute myocardial infarction," Journal of the American College of Cardiology, vol. 56, no. 9, pp. 742-743, 2010.

[13] T. Mooe, K. A. Franklin, K. Holmström, T. Rabben, and U. Wiklund, "Sleep-disordered breathing and coronary artery disease: long-term prognosis," American Journal of Respiratory and Critical Care Medicine, vol. 164, no. 10, pp. 1910-1913, 2001.

[14] S. Redline, G. Yenokyan, D. J. Gottlieb et al., "Obstructive sleep apnea-hypopnea and incident stroke: the sleep heart health study," American Journal of Respiratory and Critical Care Medicine, vol. 182, no. 2, pp. 269-277, 2010.

[15] H. K. Yaggi, J. Concato, W. N. Kernan, J. H. Lichtman, L. M. Brass, and V. Mohsenin, "Obstructive sleep apnea as a risk factor for stroke and death," The New England Journal of Medicine, vol. 353, no. 19, pp. 2034-2041, 2005.

[16] D. J. Gottlieb, G. Yenokyan, A. B. Newman et al., "Prospective study of obstructive sleep apnea and incident coronary heart disease and heart failure: the sleep heart health study," Circulation, vol. 122, no. 4, pp. 352-360, 2010.

[17] D. D. Sin, F. Fitzgerald, J. D. Parker, G. Newton, J. S. Floras, and T. D. Bradley, "Risk factors for central and obstructive sleep apnea in 450 men and women with congestive heart failure," The American Journal of Respiratory and Critical Care Medicine, vol. 160, no. 4, pp. 1101-1106, 1999.

[18] M. Badran, N. Ayas, and I. Laher, "Cardiovascular complications of sleep apnea: role of oxidative stress," Oxidative Medicine and Cellular Longevity, vol. 2014, Article ID 985258, 10 pages, 2014.

[19] P. Derentowicz, K. Markiewicz, M. Wawrzyniak, I. CzerwińskaKartowicz, E. Buława, and H. Siwińska-Gołebiowska, "Nitric oxide (NO)_nobel prize in medicine and physiology for 1998," Medycyna Wieku Rozwojowego, vol. 4, no. 2, pp. 209-217, 2000.

[20] V. Bauer and R. Sotníková, "Nitric oxide-the endotheliumderived relaxing factor and its role in endothelial functions," General Physiology and Biophysics, vol. 29, no. 4, pp. 319-340, 2010.

[21] R. M. J. Palmer, D. S. Ashton, and S. Moncada, "Vascular endothelial cells synthesize nitric oxide from L-arginine," Nature, vol. 333, no. 6174, pp. 664-666, 1988.

[22] U. Förstermann and W. C. Sessa, "Nitric oxide synthases: regulation and function," European Heart Journal, vol. 33, no. 7, pp. 829-837, 2012.

[23] C. M. Rembold, D. B. Foster, J. D. Strauss, C. J. Wingard, and J. E. Van Eyk, "cGMP-mediated phosphorylation of heat shock protein 20 may cause smooth muscle relaxation without myosin light chain dephosphorylation in swine carotid artery," Journal of Physiology, vol. 524, no. 3, pp. 865-878, 2000.

[24] S. Moncada, R. M. J. Palmer, and E. A. Higgs, "Nitric oxide: physiology, pathophysiology, and pharmacology," Pharmacological Reviews, vol. 43, no. 2, pp. 109-142, 1991.

[25] R. F. Furchgott and J. V. Zawadzki, "The obligatory role of endothelial cells in the relaxation of arterial smooth muscle by acetylcholine," Nature, vol. 288, no. 5789, pp. 373-376, 1980.

[26] S. Moncada and E. A. Higgs, "Endogenous nitric oxide: physiology, pathology and clinical relevance," European Journal of Clinical Investigation, vol. 21, no. 4, pp. 361-374, 1991.

[27] A. A. Quyyumi, N. Dakak, N. P. Andrews et al., "Nitric oxide activity in the human coronary circulation. Impact of risk factors for coronary atherosclerosis," Journal of Clinical Investigation, vol. 95, no. 4, pp. 1747-1755, 1995.

[28] S. Fiorucci, E. Antonelli, L. Santucci et al., "Gastrointestinal safety of nitric oxide-derived aspirin is related to inhibition of 
ICE-like cysteine proteases in rats," Gastroenterology, vol. 116, no. 5, pp. 1089-1106, 1999.

[29] F. Hinder, H. D. Stubbe, H. Van Aken, R. Waurick, M. Booke, and J. Meyer, "Role of nitric oxide in sepsis-associated pulmonary edema," American Journal of Respiratory and Critical Care Medicine, vol. 159, no. 1, pp. 252-257, 1999.

[30] G. R. May, P. Crook, P. K. Moore, and C. P. Page, "The role of nitric oxide as an endogenous regulator of platelet and neutrophil activation within the pulmonary circulation of the rabbit," British Journal of Pharmacology, vol. 102, no. 3, pp. 759763, 1991.

[31] R. M. Clancy, J. Leszczynska-Piziak, and S. B. Abramson, "Nitric oxide, an endothelial cell relaxation factor, inhibits neutrophil superoxide anion production via a direct action on the NADPH oxidase," The Journal of Clinical Investigation, vol. 90, no. 3, pp. 1116-1121, 1992.

[32] J. Kanner, S. Harel, and R. Granit, "Nitric oxide as an antioxidant," Archives of Biochemistry and Biophysics, vol. 289, no. 1, pp. 130-136, 1991.

[33] B. J. Zimmerman, M. B. Grisham, and D. N. Granger, "Role of oxidants in ischemia/reperfusion-induced granulocyte infiltration," The American Journal of Physiology-Gastrointestinal and Liver Physiology, vol. 258, no. 2, pp. G185-G190, 1990.

[34] Y. Kakimoto and S. Akazawa, "Isolation and identification of NG,N-G- and N-G,N'-G-dimethyl-arginine, N-epsilon-mono-, di-, and trimethyllysine, and glucosylgalactosyl- and galactosyldelta-hydroxylysine from human urine," Journal of Biological Chemistry, vol. 245, no. 21, pp. 5751-5758, 1970.

[35] J. D. Gary and S. Clarke, "RNA and protein interactions modulated by protein arginine methylation," Progress in nucleic acid research and molecular biology, vol. 61, pp. 65-131, 1998.

[36] R. H. Boger, "Asymmetric dimethylarginine, an endogenous inhibitor of nitric oxide synthase, explains the "L-arginine paradox' and acts as a novel cardiovascular risk factor," Journal of Nutrition, vol. 134, pp. 2842S-2853S, 2004.

[37] Y. Komori, G. C. Wallace, and J. M. Fukuto, "Inhibition of purified nitric oxide synthase from rat cerebellum and macrophage by L-arginine analogs," Archives of Biochemistry and Biophysics, vol. 315, no. 2, pp. 213-218, 1994.

[38] T. Teerlink, Z. Luo, F. Palm, and C. S. Wilcox, "Cellular ADMA: regulation and action," Pharmacological Research, vol. 60, no. 6, pp. 448-460, 2009.

[39] P. Bulau, D. Zakrzewicz, K. Kitowska et al., "Analysis of methylarginine metabolism in the cardiovascular system identifies the lung as a major source of ADMA," The American Journal of Physiology_Lung Cellular and Molecular Physiology, vol. 292, no. 1, pp. L18-L24, 2007.

[40] D. E. L. Wilcken, A. S. Sim, J. Wang, and X. L. Wang, "Asymmetric dimethylarginine (ADMA) in vascular, renal and hepatic disease and the regulatory role of L-arginine on its metabolism," Molecular Genetics and Metabolism, vol. 91, no. 4, pp. 308-317, 2007.

[41] J. Leiper and P. Vallance, "Biological significance of endogenous methylarginines that inhibit nitric oxide synthases," Cardiovascular Research, vol. 43, no. 3, pp. 542-548, 1999.

[42] L. J. Druhan, S. P. Forbes, A. J. Pope, C.-A. Chen, J. L. Zweier, and A. J. Cardounel, "Regulation of eNOS-derived superoxide by endogenous methylarginines," Biochemistry, vol. 47, no. 27, pp. 7256-7263, 2008.

[43] J. Strobel, M. Mieth, B. Endress et al., "Interaction of the cardiovascular risk marker asymmetric dimethylarginine (ADMA) with the human cationic amino acid transporter 1 (CAT1)," Journal of Molecular and Cellular Cardiology, vol. 53, no. 3, pp. 392-400, 2012.

[44] X. Hu, X. Xu, G. Zhu et al., "Vascular endothelial-specific dimethylarginine dimethylaminohydrolase-1-deficient mice reveal that vascular endothelium plays an important role in removing asymmetric dimethylarginine," Circulation, vol. 120, no. 22, pp. 2222-2229, 2009.

[45] D. Wang, P. S. Gill, T. Chabrashvili et al., "Isoform-specific regulation by NG,NG-dimethylarginine dimethylaminohydrolase of rat serum asymmetric dimethylarginine and vascular endothelium-derived relaxing factor/NO," Circulation Research, vol. 101, no. 6, pp. 627-635, 2007.

[46] T. Teerlink, "ADMA metabolism and clearance," Vascular Medicine, vol. 10, supplement 1, pp. S73-S81, 2005.

[47] P. Vallance, A. Leone, A. Calver, J. Collier, and S. Moncada, "Accumulation of an endogenous inhibitor of nitric oxide synthesis in chronic renal failure," The Lancet, vol. 339, no. 8793, pp. 572-575, 1992.

[48] R. H. Böger, L. M. Sullivan, E. Schwedhelm et al., "Plasma asymmetric dimethylarginine and incidence of cardiovascular disease and death in the community," Circulation, vol. 119, no. 12, pp. 1592-1600, 2009.

[49] A. J. Pope, K. Karuppiah, and A. J. Cardounel, "Role of the PRMT-DDAH-ADMA axis in the regulation of endothelial nitric oxide production," Pharmacological Research, vol. 60, no. 6, pp. 461-465, 2009.

[50] F.-M. Boisvert, J. Côté, M.-C. Boulanger, and S. Richard, "A proteomic analysis of arginine-methylated protein complexes," Molecular \& Cellular Proteomics, vol. 2, no. 12, pp. 1319-1330, 2003.

[51] F. Abbasi, T. Asagmi, J. P. Cooke et al., "Plasma concentrations of asymmetric dimethylarginine are increased in patients with type 2 diabetes mellitus," American Journal of Cardiology, vol. 88, no. 10, pp. 1201-1203, 2001.

[52] L. Yang, Q. Xiufen, S. Shuqin et al., "Asymmetric dimethylarginine concentration and recurrence of atrial tachyarrythmias after catheter ablation in patients with persistent atrial fibrillation," Journal of Interventional Cardiac Electrophysiology, vol. 32, no. 2, pp. 147-154, 2011.

[53] J. T. Kielstein, S. M. Bode-Böger, G. Klein, S. Graf, H. Haller, and D. Fliser, "Endogenous nitric oxide synthase inhibitors and renal perfusion in patients with heart failure," European Journal of Clinical Investigation, vol. 33, no. 5, pp. 370-375, 2003.

[54] M. T. Bedford, "Arginine methylation at a glance," Journal of Cell Science, vol. 120, no. 24, pp. 4243-4246, 2007.

[55] S. W. Bae, M. C. Stühlinger, H. S. Yoo et al., "Plasma asymmetric dimethylarginine concentrations in newly diagnosed patients with acute myocardial infarction or unstable angina pectoris during two weeks of medical treatment," The American Journal of Cardiology, vol. 95, no. 6, pp. 729-733, 2005.

[56] C. Zoccali, F. Mallamaci, R. Maas et al., "Left ventricular hypertrophy, cardiac remodeling and asymmetric dimethylarginine (ADMA) in hemodialysis patients," Kidney International, vol. 62, no. 1, pp. 339-345, 2002.

[57] P. Wanby, T. Teerlink, L. Brudin et al., "Asymmetric dimethylarginine (ADMA) as a risk marker for stroke and TIA in a Swedish population," Atherosclerosis, vol. 185, no. 2, pp. 271-277, 2006.

[58] V.-P. Valkonen, H. Päivä, J. T. Salonen et al., "Risk of acute coronary events and serum concentration of asymmetrical 
dimethylarginine," The Lancet, vol. 358, no. 9299, pp. 2127-2128, 2001.

[59] Y. Cao, K. Yang, Z. Zhang, M. Ouyang, and L. Xiao, "Correlation between plasma asymmetric dimethylarginine and different types of coronary heart disease," Zhong Nan Da Xue Xue Bao Yi Xue Ban, vol. 35, no. 4, pp. 301-306, 2010.

[60] Z. Shao, Z. Wang, K. Shrestha et al., "Pulmonary hypertension associated with advanced systolic heart failure: dysregulated arginine metabolism and importance of compensatory dimethylarginine dimethylaminohydrolase-1," Journal of the American College of Cardiology, vol. 59, no. 13, pp. 1150-1158, 2012.

[61] A. Barceló, M. de la Peña, O. Ayllón et al., "Increased plasma levels of asymmetric dimethylarginine and soluble CD40 ligand in patients with sleep apnea," Respiration, vol. 77, no. 1, pp. 8590, 2009.

[62] Y. Ozkan, H. Firat, B. Şimşek, M. Torun, and S. Yardim-Akaydin, "Circulating nitric oxide (NO), asymmetric dimethylarginine (ADMA), homocysteine, and oxidative status in obstructive sleep apnea-hypopnea syndrome (OSAHS)," Sleep and Breathing, vol. 12, no. 2, pp. 149-154, 2008.

[63] Y. Ohike, K. Kozaki, K. Iijima et al., "Amelioration of vascular endothelial dysfunction in obstructive sleep apnea syndrome by nasal continuous positive airway pressure: possible involvement of nitric oxide and asymmetric NG, NG-dimethylarginine," Circulation Journal, vol. 69, no. 2, pp. 221-226, 2005.

[64] K. Sugamura and J. F. Keaney Jr., "Reactive oxygen species in cardiovascular disease," Free Radical Biology and Medicine, vol. 51, no. 5, pp. 978-992, 2011.

[65] M. Li, X. W. Zhang, W. S. Hou, and Z. Y. Tang, "Insomnia and risk of cardiovascular disease: a meta-analysis of cohort studies," International Journal of Cardiology, vol. 176, pp. 1044-1047, 2014.

[66] D. Praticò, C. M. Clark, F. Liun, V. Y. M. Lee, and J. Q. Trojanowski, "Increase of brain oxidative stress in mild cognitive impairment: a possible predictor of Alzheimer disease," Archives of Neurology, vol. 59, no. 6, pp. 972-976, 2002.

[67] R. Schnabel and S. Blankenberg, "Oxidative stress in cardiovascular disease: successful translation from bench to bedside?" Circulation, vol. 116, no. 12, pp. 1338-1340, 2007.

[68] E. Cadenas and K. J. A. Davies, "Mitochondrial free radical generation, oxidative stress, and aging," Free Radical Biology and Medicine, vol. 29, no. 3-4, pp. 222-230, 2000.

[69] L. Lavie, "Oxidative stress in obstructive sleep apnea and intermittent hypoxia-revisited - the bad ugly and good: implications to the heart and brain," Sleep Medicine Reviews, vol. 20, pp. 27-45, 2015.

[70] P. W. M. Kleikers, K. Wingler, J. J. R. Hermans et al., "NADPH oxidases as a source of oxidative stress and molecular target in ischemia/reperfusion injury," Journal of Molecular Medicine, vol. 90, no. 12, pp. 1391-1406, 2012.

[71] J. Duranteau, N. S. Chandel, A. Kulisz, Z. Shao, and P. T. Schumacker, "Intracellular signaling by reactive oxygen species during hypoxia in cardiomyocytes," The Journal of Biological Chemistry, vol. 273, no. 19, pp. 11619-11624, 1998.

[72] M. Valko, D. Leibfritz, J. Moncol, M. T. D. Cronin, M. Mazur, and J. Telser, "Free radicals and antioxidants in normal physiological functions and human disease," International Journal of Biochemistry and Cell Biology, vol. 39, no. 1, pp. 44-84, 2007.

[73] A. D. McGown, H. Makker, C. Elwell, P. G. Al Rawi, A. Valipour, and S. G. Spiro, "Measurement of changes in cytochrome oxidase redox state during obstructive sleep apnea using nearinfrared spectroscopy," Sleep, vol. 26, no. 6, pp. 710-716, 2003.
[74] P. S. Stl and B. Johansson, "Abnormal mitochondria organization and oxidative activity in the palate muscles of long-term snorers with obstructive sleep apnea," Respiration, vol. 83, no. 5, pp. 407-417, 2012.

[75] X. Shan, L. Chi, Y. Ke et al., "Manganese superoxide dismutase protects mouse cortical neurons from chronic intermittent hypoxia-mediated oxidative damage," Neurobiology of Disease, vol. 28, no. 2, pp. 206-215, 2007.

[76] N. Wang, S. A. Khan, N. R. Prabhakar, and J. Nanduri, "Impairment of pancreatic $\beta$-cell function by chronic intermittent hypoxia," Experimental Physiology, vol. 98, no. 9, pp. 1376-1385, 2013.

[77] R. Schulz, S. Mahmoudi, K. Hattar et al., "Enhanced release of superoxide from polymorphonuclear neutrophils in obstructive sleep apnea: impact of continuous positive airway pressure therapy," American Journal of Respiratory and Critical Care Medicine, vol. 162, no. 2, pp. 566-570, 2000.

[78] L. Dyugovskaya, P. Lavie, and L. Lavie, "Increased adhesion molecules expression and production of reactive oxygen species in leukocytes of sleep apnea patients," The American Journal of Respiratory and Critical Care Medicine, vol. 165, no. 7, pp. 934939, 2002.

[79] L. Dyugovskaya, A. Polyakov, P. Lavie, and L. Lavie, "Delayed neutrophil apoptosis in patients with sleep apnea," American Journal of Respiratory and Critical Care Medicine, vol. 177, no. 5, pp. 544-554, 2008.

[80] L. Loffredo, A. M. Zicari, F. Occasi et al., "Endothelial dysfunction and oxidative stress in children with sleep disordered breathing: role of NADPH oxidase," Atherosclerosis, vol. 240, no. 1, pp. 222-227, 2015.

[81] Y.-J. Peng, J. Nanduri, G. Yuan et al., "NADPH oxidase is required for the sensory plasticity of the carotid body by chronic intermittent hypoxia," Journal of Neuroscience, vol. 29, no. 15, pp. 4903-4910, 2009.

[82] G. Zhan, F. Serrano, P. Fenik et al., "NADPH oxidase mediates hypersomnolence and brain oxidative injury in a murine model of sleep apnea," American Journal of Respiratory and Critical Care Medicine, vol. 172, no. 7, pp. 921-929, 2005.

[83] J. M. Dopp, N. R. Philippi, N. J. Marcus et al., "Xanthine oxidase inhibition attenuates endothelial dysfunction caused by chronic intermittent hypoxia in rats," Respiration, vol. 82, no. 5, pp. 458467, 2011.

[84] A. A. El Solh, R. Saliba, T. Bosinski, B. J. B. Grant, E. Berbary, and N. Miller, "Allopurinol improves endothelial function in sleep apnoea: a randomised controlled study," European Respiratory Journal, vol. 27, no. 5, pp. 997-1002, 2006.

[85] L. Lavie, "Oxidative stress inflammation and endothelial dysfunction in obstructive sleep apnea," Frontiers in BioscienceElite, vol. 4, no. 4, pp. 1391-1403, 2012.

[86] M. del Ben, M. Fabiani, L. Loffredo et al., "Oxidative stress mediated arterial dysfunction in patients with obstructive sleep apnoea and the effect of continuous positive airway pressure treatment," BMC Pulmonary Medicine, vol. 12, article 36, 2012.

[87] L. Lavie, A. Vishnevsky, and P. Lavie, "Evidence for lipid peroxidation in obstructive sleep apnea," Sleep, vol. 27, no. 1, pp. 123-128, 2004.

[88] A. Svatikova, R. Wolk, L. O. Lerman et al., "Oxidative stress in obstructive sleep apnoea," European Heart Journal, vol. 26, no. 22, pp. 2435-2439, 2005.

[89] E. Vatansever, E. Surmen-Gur, A. Ursavas, and M. Karadag, "Obstructive sleep apnea causes oxidative damage to plasma 
lipids and proteins and decreases adiponectin levels," Sleep and Breathing, vol. 15, no. 3, pp. 275-282, 2011.

[90] M. Yamauchi, H. Nakano, J. Maekawa et al., "Oxidative stress in obstructive sleep apnea," Chest, vol. 127, no. 5, pp. 1674-1679, 2005.

[91] R. Schulz, D. Schmidt, A. Blum et al., "Decreased plasma levels of nitric oxide derivatives in obstructive sleep apnoea: response to CPAP therapy," Thorax, vol. 55, no. 12, pp. 1046-1051, 2000.

[92] M. Kato, P. Roberts-Thomson, B. G. Phillips et al., "Impairment of endothelium-dependent vasodilation of resistance vessels in patients with obstructive sleep apnea," Circulation, vol. 102, no. 21, pp. 2607-2610, 2000.

[93] M. S. M. Ip, B. Lam, L.-Y. Chan et al., "Circulating nitric oxide is suppressed in obstructive sleep apnea and is reversed by nasal continuous positive airway pressure," American Journal of Respiratory and Critical Care Medicine, vol. 162, no. 6, pp. 21662171, 2000.

[94] L. Lavie, A. Hefetz, R. Luboshitzky, and P. Lavie, "Plasma levels of nitric oxide and L-arginine in sleep apnea patients: effects of nCPAP treatment," Journal of Molecular Neuroscience, vol. 21, no. 1, pp. 57-63, 2003.

[95] R. Muñoz-Hernandez, A. J. Vallejo-Vaz, A. Sanchez Armengol et al., "Obstructive sleep apnoea syndrome, endothelial function and markers of endothelialization. Changes after CPAP," PLOS ONE, vol. 10, Article ID e0122091, 2015.

[96] A. Svatikova, R. Wolk, H. H. Wang et al., "Circulating free nitrotyrosine in obstructive sleep apnea," American Journal of Physiology-Regulatory Integrative and Comparative Physiology, vol. 287, no. 2, pp. R284-R287, 2004.

[97] M. A. Tabrizi-Fard, T. S. Maurer, and H.-L. Fung, "In vivo disposition of 3-nitro-L-tyrosine in rats: implications on tracking systemic peroxynitrite exposure," Drug Metabolism and Disposition, vol. 27, no. 4, pp. 429-431, 1999.

[98] B. T. Patt, D. Jarjoura, D. N. Haddad et al., "Endothelial dysfunction in the microcirculation of patients with obstructive sleep apnea," American Journal of Respiratory and Critical Care Medicine, vol. 182, no. 12, pp. 1540-1545, 2010.

[99] S. Jelic and T. H. le Jemtel, "Inflammation, oxidative stress, and the vascular endothelium in obstructive sleep apnea," Trends in Cardiovascular Medicine, vol. 18, no. 7, pp. 253-260, 2008.

[100] T. Tanaka, H. Nakamura, J. Yodoi, and E. T. Bloom, "Redox regulation of the signaling pathways leading to eNOS phosphorylation," Free Radical Biology and Medicine, vol. 38, no. 9, pp. 1231-1242, 2005.

[101] U. A. Arnet, A. McMillan, J. L. Dinerman, B. Ballermann, and C. J. Lowenstein, "Regulation of endothelial nitric-oxide synthase during hypoxia," Journal of Biological Chemistry, vol. 271, no. 25, pp. 15069-15073, 1996.

[102] M. Shirai, J. T. Pearson, A. Shimouchi et al., "Changes in functional and histological distributions of nitric oxide synthase caused by chronic hypoxia in rat small pulmonary arteries," British Journal of Pharmacology, vol. 139, no. 5, pp. 899-910, 2003.

[103] M. Takemoto, J. Sun, J. Hiroki, H. Shimokawa, and J. K. Liao, "Rho-kinase mediates hypoxia-induced downregulation of endothelial nitric oxide synthase," Circulation, vol. 106, no. 1, pp. 57-62, 2002.

[104] M. Toporsian, K. Govindaraju, M. Nagi, D. Eidelman, G. Thibault, and M. E. Ward, "Downregulation of endothelial nitric oxide synthase in rat aorta after prolonged hypoxia in vivo," Circulation Research, vol. 86, no. 6, pp. 671-675, 2000.
[105] T. Murata, H. Yamawaki, M. Hori, K. Sato, H. Ozaki, and H. Karaki, "Hypoxia impairs endothelium-dependent relaxation in organ cultured pulmonary artery," European Journal of Pharmacology, vol. 421, no. 1, pp. 45-53, 2001.

[106] E. Kaczmarek, J. P. Bakker, D. N. Clarke et al., "Molecular biomarkers of vascular dysfunction in obstructive sleep apnea," PLoS ONE, vol. 8, no. 7, Article ID e70559, 2013.

[107] C. Antoniades, C. Shirodaria, N. Warrick et al., "5Methyltetrahydrofolate rapidly improves endothelial function and decreases superoxide production in human vessels: effects on vascular tetrahydrobiopterin availability and endothelial nitric oxide synthase coupling," Circulation, vol. 114, no. 11, pp. 1193-1201, 2006.

[108] S. Varadharaj, K. Porter, A. Pleister et al., "Endothelial nitric oxide synthase uncoupling: a novel pathway in OSA induced vascular endothelial dysfunction," Respiratory Physiology \& Neurobiology, vol. 207, pp. 40-47, 2015.

[109] Y. Bai, L. Sun, L. Du et al., "Association of circulating levels of asymmetric dimethylarginine (ADMA) with carotid intimamedia thickness: evidence from 6168 participants," Ageing Research Reviews, vol. 12, no. 2, pp. 699-707, 2013.

[110] F. Perticone, A. Sciacqua, R. Maio et al., "Asymmetric dimethylarginine, L-arginine, and endothelial dysfunction in essential hypertension," Journal of the American College of Cardiology, vol. 46, no. 3, pp. 518-523, 2005.

[111] M. I. Yilmaz, A. Sonmez, M. Saglam et al., "ADMA levels correlate with proteinuria, secondary amyloidosis, and endothelial dysfunction," Journal of the American Society of Nephrology, vol. 19, no. 2, pp. 388-395, 2008.

[112] H. Päivä, M. Kähönen, T. Lehtimäki et al., "Levels of asymmetrical dimethylarginine are predictive of brachial artery flowmediated dilation 6 years later. The Cardiovascular Risk in Young Finns Study," Atherosclerosis, vol. 212, no. 2, pp. 512-515, 2010.

[113] S. Nural, E. Günay, B. Halici, S. Celik, and M. Ünlü, "Inflammatory processes and effects of continuous positive airway pressure (CPAP) in overlap syndrome," Inflammation, vol. 36, no. 1, pp. 66-74, 2013.

[114] A. Barcelo, J. Piérola, M. de la Peña et al., "Day-night variations in endothelial dysfunction markers and haemostatic factors in sleep apnoea," European Respiratory Journal, vol. 39, no. 4, pp. 913-918, 2012.

[115] D. Gozal, L. Kheirandish-Gozal, L. D. Serpero, O. S. Capdevila, and E. Dayyat, "Obstructive sleep apnea and endothelial function in school-aged nonobese children: effect of adenotonsillectomy," Circulation, vol. 116, no. 20, pp. 2307-2314, 2007.

[116] R. H. Böger and E. S. Ron, "L-arginine improves vascular function by overcoming the deleterious effects of ADMA, a novel cardiovascular risk factor," Alternative Medicine Review, vol. 10, no. 1, pp. 14-23, 2005.

[117] J. P. F. Chin-Dusting, C. T. Alexander, P. J. Arnold, W. C. Hodgson, A. S. Lux, and G. L. R. Jennings, "Effects of in vivo and in vitro L-arginine supplementation on healthy human vessels," Journal of Cardiovascular Pharmacology, vol. 28, no. 1, pp. 158$166,1996$.

[118] S. P. Schulman, L. C. Becker, D. A. Kass et al., "L-arginine therapy in acute myocardial infarction: the Vascular Interaction with Age in Myocardial Infarction (VINTAGE MI) Randomized Clinical Trial," Journal of the American Medical Association, vol. 295, no. 1, pp. 58-64, 2006. 
[119] J. Bełtowski and A. Kedra, "Asymmetric dimethylarginine (ADMA) as a target for pharmacotherapy," Pharmacological Reports, vol. 58, no. 2, pp. 159-178, 2006.

[120] Y. T. Ghebremariam, D. A. Erlanson, and J. P. Cooke, "A novel and potent inhibitor of dimethylarginine dimethylaminohydrolase: a modulator of cardiovascular nitric oxide," Journal of Pharmacology and Experimental Therapeutics, vol. 348, no. 1, pp. 69-76, 2014.

[121] M. Nandi, P. Kelly, B. Torondel et al., "Genetic and pharmacological inhibition of dimethylarginine dimethylaminohydrolase 1 is protective in endotoxic shock," Arteriosclerosis, Thrombosis, and Vascular Biology, vol. 32, no. 11, pp. 2589-2597, 2012. 


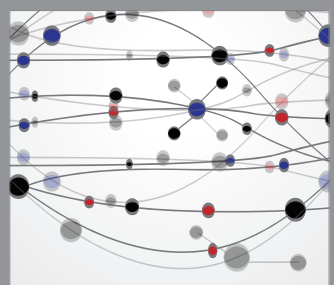

The Scientific World Journal
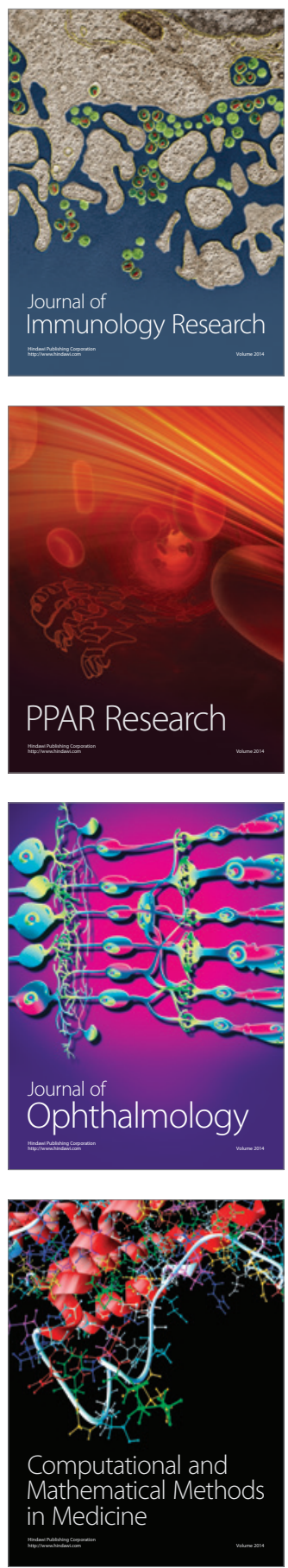

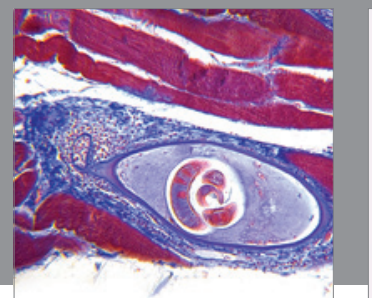

Gastroenterology

Research and Practice
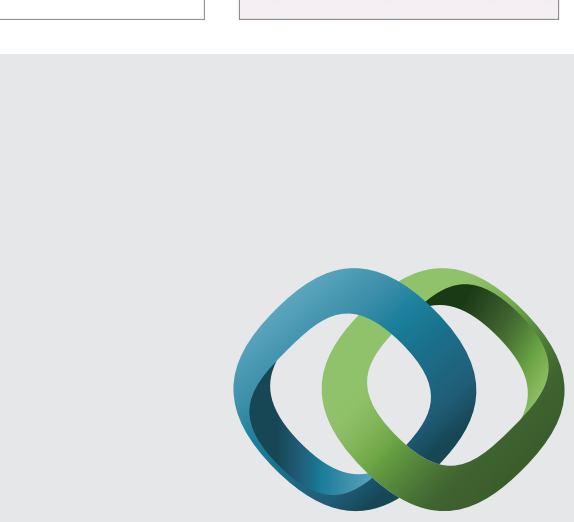

\section{Hindawi}

Submit your manuscripts at

http://www.hindawi.com
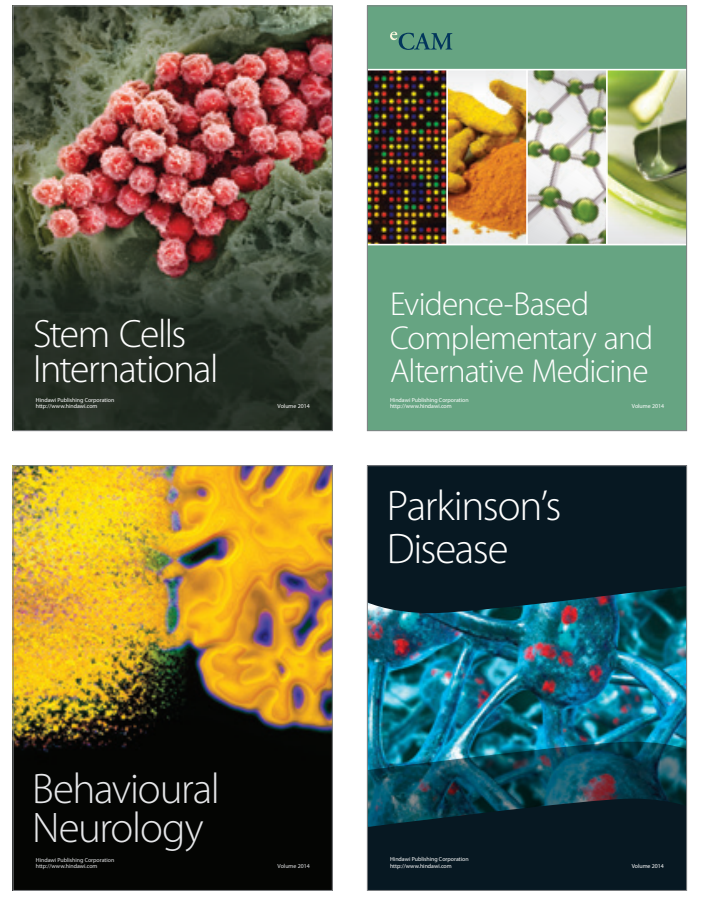
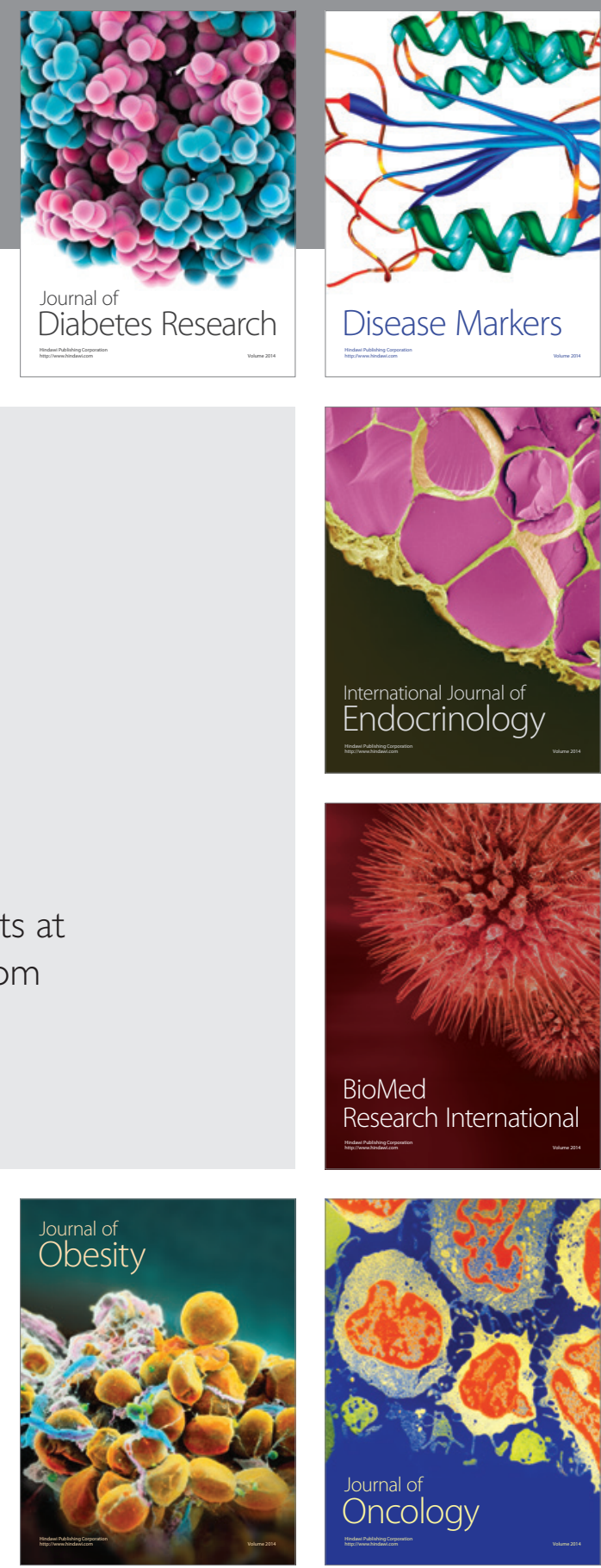

Disease Markers
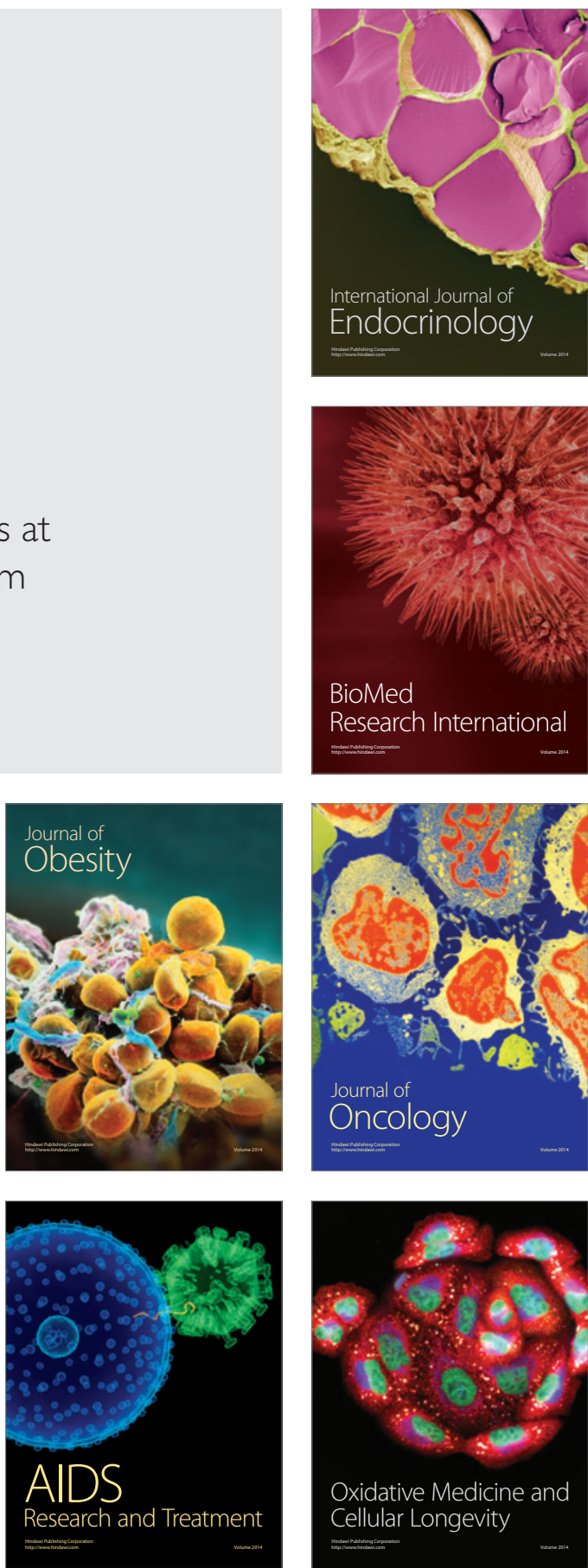